\title{
EXPLICIT CAYLEY TRIPLES IN REAL FORMS OF $G_{2}, F_{4}$, AND $E_{6}$
}

\author{
DRAGOMIR Ž. ĐoKOVIĆ
}

\begin{abstract}
We re-examine the problem of classifying the nilpotent adjoint orbits in semisimple real Lie algebras. We present the list of representatives of these orbits, as well as those of related Cayley triples, in the case when the complexification of the algebra is one of the exceptional complex Lie algebras mentioned in the title.
\end{abstract}

\section{Introduction.}

The nilpotent adjoint orbits in non-compact real forms $\mathfrak{g}$ of exceptional complex Lie algebras $\mathfrak{g}^{c}$ have been classified in our papers $[6,7]$. That classification is indirect because it makes use of the Sekiguchi bijection (see the next section). Hence our classification is not as explicit as one would like it to be. For instance the list of representatives of the nilpotent adjoint orbits of $\mathfrak{g}$ is missing. The main objective of this paper is the fill this gap when $\mathfrak{g}^{c}$ is of the type $G_{2}, F_{4}$, or $E_{6}$.

Let $\mathfrak{g}$ be an arbitrary semisimple real Lie algebra (of finite dimension). Let $\mathfrak{g}^{c}$ be the complexification of $\mathfrak{g}$, and $G$ (resp. $G^{c}$ ) the adjoint group of $\mathfrak{g}\left(\right.$ resp. $\mathfrak{g}^{c}$ ). We fix a Cartan decomposition $\mathfrak{g}=\mathfrak{k} \oplus \mathfrak{p}$ of $\mathfrak{g}$, with Cartan involution $\theta$ (extended also to $\mathfrak{g}^{c}$ ). Let $K^{c}$ be the connected Lie subgroup of $G^{c}$ corresponding to $\mathfrak{k}^{c}$.

In Section 2 we describe the Sekiguchi bijection which gives the one-to-one correspondence between the nonzero nilpotent $G$-orbits in $\mathfrak{g}$ and the nonzero nilpotent $K^{c}$-orbits in $\mathfrak{p}^{c}$.

In Section 3 we sketch a new approach to the problem of classification of nilpotent adjoint orbits in $\mathfrak{g}$. By using a theorem from our note [9], we are able to select from the list of the nilpotent $G^{c}$-orbits $\mathcal{O} \subset \mathfrak{g}^{c}$ those orbits that possess real points, i.e., such that $\mathcal{O} \cap \mathfrak{g} \neq \emptyset$. Theorem 2 provides a method of identifying the $G$-orbits into which $\mathcal{O} \cap \mathfrak{g}$ splits.

In Section 4 we construct Chevalley systems for $\mathfrak{g}^{c}$ when the latter is of type $G_{2}, F_{4}$, or $E_{6}$. We also describe the action of the conjugation $\sigma$ corresponding to the real form $\mathfrak{g} \subset \mathfrak{g}^{c}$. These data are necessary if one wants to list the representatives of the nilpotent adjoint orbits in $\mathfrak{g}$. 
Finally, in Section 5 we list the representatives $(E, H, F)$ of the $G$-orbits of the real Cayley triples (defined in Section 2) in $\mathfrak{g}$ for each of the noncompact real forms of $G_{2}, F_{4}$, and $E_{6}$. The elements $E$ listed there are the representatives of the nonzero nilpotent $G$-orbits in $\mathfrak{g}$.

The following misprints have been detected in our paper [6]:

p. 511, Table VIII, orbit 1: Replace $\mathfrak{s u}(3)$ with $\mathfrak{s u}(4)$.

p. 514, Table XI, orbit 85: Replace 1 in column 6 with 7.

p. 516, Table XIII, orbit 16: Replace $\mathfrak{s u}(7)$ with $\mathfrak{s o}(7)$.

p. 520, Table XV, orbit 11: Replace 62 in column 4 with 63.

We also mention that in $[4]$ (where some of the results of $[6,7]$ are quoted) the Dynkin diagram of $F_{4}$, in the table of nilpotent orbits in $E_{6(-26)}$ on p. 152 , should have the arrow pointing in the other direction.

\section{Cayley transformation and Sekiguchi bijection.}

Let $\mathfrak{g}$ be a real semisimple Lie algebra and $\mathfrak{g}^{c}$ its complexification. We fix a Cartan involution $\theta$ of $\mathfrak{g}$ and set $\mathfrak{k}=(1+\theta) \mathfrak{g}$ and $\mathfrak{p}=(1-\theta) \mathfrak{g}$. Hence $\mathfrak{g}=\mathfrak{k} \oplus \mathfrak{p}$ is the Cartan decomposition of $\mathfrak{g}$. We denote by $G$ the adjoint group of $\mathfrak{g}$ and by $K$ the connected Lie subgroup of $G$ corresponding to $\mathfrak{k}$. Similarly, $G^{c}$ will denote the adjoint group of $\mathfrak{g}^{c}$ and $K^{c}$ its connected Lie subgroup corresponding to $\mathfrak{k}^{c}$.

The involution $\theta$ of $\mathfrak{g}$ extends uniquely to an involutive automorphism of $\mathfrak{g}^{c}$ which we also denote by $\theta$. Let $\sigma$ be the conjugation of $\mathfrak{g}^{c}$ defined by $\mathfrak{g}$. Thus we have $\sigma(X)=X$ and $\sigma(i X)=-i X$ for $X \in \mathfrak{g}$. Then $\sigma \theta=\theta \sigma$ and $\sigma_{u}:=\theta \sigma$ is the conjugation of $\mathfrak{g}^{c}$ corresponding to the compact real form $\mathfrak{g}_{u}=\mathfrak{k} \oplus i \mathfrak{p}$ of $\mathfrak{g}^{c}$.

We say that $(E, H, F)$ is a standard triple if $\{E, H, F\} \subset \mathfrak{g}^{c}, E \neq 0$, and they satisfy the relations

$$
[H, E]=2 E,[H, F]=-2 F,[F, E]=H .
$$

By adopting the terminology of [4], we say that $H$ is the neutral, E nilpositive, and $F$ nilnegative element of this triple. (Our definition of standard triples is different from the one in [4] where the last relation above is replaced with $[E, F]=H$.)

We say that a standard triple $(E, H, F)$ is a real Cayley triple if $E, H, F$ belong to $\mathfrak{g}$ and $\theta(E)=F$. In that case we have $\theta(H)=-H$, i.e., $H \in \mathfrak{p}$.

A standard triple $(E, H, F)$ is called normal if $H \in \mathfrak{k}^{c}$ and $E, F \in \mathfrak{p}^{c}$. We say that a normal triple $(E, H, F)$ is a complex Cayley triple if $\sigma(E)=-F$.

Let $(E, H, F)$ be a real Cayley triple and write $E=U+V$ with $U \in \mathfrak{k}$ 
and $V \in \mathfrak{p}$. Since $F=\theta(E)$, we have $F=U-V$. It follows easily that

$$
[H, U]=2 V,[H, V]=2 U, H=2[U, V] .
$$

If we define the elements:

$$
\begin{gathered}
E^{\prime}=\frac{1}{2}(H+i F-i E)=\frac{1}{2} H-i V, \\
H^{\prime}=i(E+F)=2 i U, \\
F^{\prime}=\frac{1}{2}(-H+i F-i E)=-\frac{1}{2} H-i V,
\end{gathered}
$$

then $\left(E^{\prime}, H^{\prime}, F^{\prime}\right)$ is a complex Cayley triple and we refer to the map

$$
(E, H, F) \mapsto\left(E^{\prime}, H^{\prime}, F^{\prime}\right)
$$

as the Cayley transformation. We also say that $\left(E^{\prime}, H^{\prime}, F^{\prime}\right)$ is the Cayley transform of $(E, H, F)$.

The inverse $\left(E^{\prime}, H^{\prime}, F^{\prime}\right) \mapsto(E, H, F)$ of the Cayley transformation is given by the formulae

$$
U=-\frac{i}{2} H^{\prime}, H=E^{\prime}-F^{\prime}, V=\frac{i}{2}\left(E^{\prime}+F^{\prime}\right)
$$

i.e.,

$$
E=\frac{i}{2}\left(-H^{\prime}+E^{\prime}+F^{\prime}\right), \quad H=E^{\prime}-F^{\prime}, \quad F=-\frac{i}{2}\left(H^{\prime}+E^{\prime}+F^{\prime}\right) .
$$

For an element $X \in \mathfrak{g}$ we say that it is nilpotent if the linear operator $\operatorname{ad}(X)$ is nilpotent. The group $G\left(\right.$ resp. $\left.G^{c}\right)$ acts on $\mathfrak{g}\left(\right.$ resp. $\left.\mathfrak{g}^{c}\right)$ via the adjoint representation.

Let $\mathcal{O}$ be a nonzero nilpotent $G$-orbit in $\mathfrak{g}$. Choose $E_{0} \in \mathcal{O}$. By JacobsonMorozov Theorem, there exist $H_{0}, F_{0} \in \mathfrak{g}$ such that $\left(E_{0}, H_{0}, F_{0}\right)$ is a standard triple. By [4, Theorem 9.4.1] there exists $g \in G$ such that $g \cdot\left(E_{0}, H_{0}, F_{0}\right)=$ $(E, H, F)$ is a real Cayley triple. Let $\left(E^{\prime}, H^{\prime}, F^{\prime}\right)$ be the Cayley transform of $(E, H, F)$. Finally let $\mathcal{O}^{\prime}:=K^{c} \cdot E^{\prime}$ be the nilpotent $K^{c}$-orbit in $\mathfrak{p}^{c}$ through $E^{\prime}$. The map that assigns $\mathcal{O}^{\prime}$ to $\mathcal{O}$ is well defined and it was shown by J. Sekiguchi [16] and the author [5] that it establishes a bijection between the nonzero nilpotent $G$-orbits in $\mathfrak{g}$ and the nonzero nilpotent $K^{c}$-orbits in $\mathfrak{p}^{c}$. Following [4], we shall refer to this map as the Sekiguchi bijection. 


\section{An approach to the classification of nilpotent $G$-orbits in $\mathfrak{g}$.}

Let $\mathfrak{a}$ be a maximal abelian subspace of $\mathfrak{p}$, and $\mathfrak{m}$ the centralizer of $\mathfrak{a}$ in $\mathfrak{k}$. Let $\mathfrak{h}$ be a Cartan subalgebra of $\mathfrak{g}$ such that $\mathfrak{h} \supset \mathfrak{a}$. Then

$$
\mathfrak{h}=\mathfrak{a} \oplus(\mathfrak{h} \cap \mathfrak{m})
$$

and $\mathfrak{h} \cap \mathfrak{m}$ is a Cartan subalgebra of $\mathfrak{m}$. We recall that $\mathfrak{m}$ is reductive in $\mathfrak{k}$. The complexification $\mathfrak{h}^{c}$ is a Cartan subalgebra of $\mathfrak{g}^{c}$.

Let $\Phi$ be the root system of $\left(\mathfrak{g}^{c}, \mathfrak{h}^{c}\right)$. Each root $\alpha \in \Phi$ is a complex linear functional on $\mathfrak{h}^{c}$ which takes real values on the real form

$$
\mathfrak{h}_{0}:=\mathfrak{a} \oplus i(\mathfrak{h} \cap \mathfrak{m})
$$

of $\mathfrak{h}^{c}$. Let $\rho(\alpha)$ denote the restriction of $\alpha \in \Phi$ to the subspace $\mathfrak{a}$. We set

$$
\Phi_{0}:=\{\alpha \in \Phi: \rho(\alpha)=0\}
$$

and $\Phi_{1}:=\Phi \backslash \Phi_{0}$. The set

$$
\Sigma:=\left\{\rho(\alpha): \alpha \in \Phi_{1}\right\}
$$

is a root system (not necessarily reduced) in the dual space of $\mathfrak{a}$.

We choose a base $\Pi$ of $\Phi$ containing a base $\Pi_{0}$ of $\Phi_{0}$, and we set $\Pi_{1}:=$ $\Pi \backslash \Pi_{0}$. Then the set

$$
\Theta:=\left\{\rho(\alpha): \alpha \in \Pi_{1}\right\}
$$

is a base of $\Sigma$.

Let $C_{\Pi} \subset \mathfrak{h}_{0}$ (resp. $C_{\Theta} \subset \mathfrak{a}$ ) be the closed fundamental Weyl chamber of $\Phi$ (resp. $\Sigma$ ) corresponding to the base $\Pi$ (resp. $\Theta$ ). Note that $C_{\Theta}=\mathfrak{a} \cap C_{\Pi}$.

Let $\mathcal{O} \subset \mathfrak{g}^{c}$ be a nonzero nilpotent $G^{c}$-orbit. Then we can choose a standard triple $(E, H, F)$ such that $E \in \mathcal{O}$ and $H \in C_{\Pi}$. The neutral element $H$ of this triple is uniquely determined by $\mathcal{O}$ and is called the characteristic of $\mathcal{O}$. One usually identifies $H$ by means of the labels $\alpha(H), \alpha \in \Pi$. Hence the Dynkin diagram of $(\Phi, \Pi)$ together with the labels $\alpha(H), \alpha \in \Pi$, determines uniquely the orbit $\mathcal{O}$. All the labels $\alpha(H), \alpha \in \Pi$, belong to $\{0,1,2\}$.

In the case where $\mathfrak{g}^{c}$ is one of the five simple exceptional Lie algebras $G_{2}, F_{4}, E_{6}, E_{7}$, or $E_{8}$, one can find the list of nonzero nilpotent orbits, i.e, the corresponding labelled Dynkin diagrams in many places, eg. $[3,4,10,11]$. In the case when $\mathfrak{g}^{c}$ is a simple classical Lie algebra see [4].

Theorem 2 of [9], when specialized to the real field $\mathbf{R}$, gives the following result. 
Theorem 1. Let $\mathcal{O} \subset \mathfrak{g}^{c}$ be a nonzero nilpotent $G^{c}$-orbit and $H \in C_{\Pi}$ its characteristic. Then $\mathcal{O} \cap \mathfrak{g} \neq \emptyset$ if and only if $H \in C_{\Theta}$.

The condition $H \in C_{\Theta}$ can be verified easily by using the Satake diagram of $G$. Recall that the Satake diagram is obtained from the Dynkin diagram of $(\Phi, \Pi)$ by colouring in black all vertices in $\Pi_{0}$ and by joining by a curved arrow two white vertices $\alpha, \beta \in \Pi_{1}$ whenever $\rho(\alpha)=\rho(\beta)$. The condition $H \in C_{\Theta}$ is satisfied if and only if all the weights $\alpha(H)=0$ for $\alpha \in \Pi_{0}$ and $\alpha(H)=\beta(H)$ whenever $\alpha, \beta \in \Pi_{1}$ are joined by a curved arrow.

Let $\mathcal{O} \subset \mathfrak{g}^{c}$ be a nonzero nilpotent $G^{c}$-orbit, and let $H \in C_{\Theta}$ be its characteristic. Then $H$ defines the $\mathbf{Z}$-gradation

$$
\mathfrak{g}=\oplus_{k \in \mathbf{Z}} \mathfrak{g}(k)
$$

with

$$
\mathfrak{g}(k):=\{x \in \mathfrak{g}:[H, X]=k X\} .
$$

Let $G(0)^{c}$ (resp. $G(0)$ ) be the connected Lie subgroup of $G^{c}$ (resp. $G$ ) corresponding to the subalgebra $\mathfrak{g}(0)^{c} \subset \mathfrak{g}^{c}$ (resp. $\mathfrak{g}(0) \subset \mathfrak{g}$ ). The pair $\left(G(0)^{c}, \mathfrak{g}(2)^{c}\right)$ is a prehomogeneous vector space, i.e., there exists a unique $G(0)^{c}$-orbit, say $\mathcal{O}(2)^{c}$, in $\mathfrak{g}(2)^{c}$ which is open (and dense) in $\mathfrak{g}(2)^{c}$. Moreover we have $\mathcal{O}(2)^{c}=\mathcal{O} \cap \mathfrak{g}(2)^{c}$. It is known [12, Lemma 5] that the centralizer $Z_{G^{c}}(H)$ is connected, and so it coincides with $G(0)^{c}$. Consequently, we have $Z_{G}(H)=G \cap G(0)^{c}$ and $G(0)$ is the identity component of $Z_{G}(H)$.

Let $\mathcal{O}(2):=\mathcal{O}(2)^{c} \cap \mathfrak{g}(2)$. This is a nonempty Zariski open subset of $\mathfrak{g}(2)$, and so it has only finitely many connected components in the Euclidean topology. Each of these components is a $G(0)$-orbit.

Theorem 2. The map which assigns to a $Z_{G}(H)$-orbit $\mathcal{O}_{1} \subset \mathcal{O}(2)$ the $G$-orbit containing $\mathcal{O}_{1}$ is a bijection from the set of $Z_{G}(H)$-orbits in $\mathcal{O}(2)$ to the set of $G$-orbits in $\mathcal{O} \cap \mathfrak{g}$.

Proof. Let $E \in \mathcal{O} \cap \mathfrak{g}$. By Jacobson-Morozov theorem there exist $H^{\prime}, F \in \mathfrak{g}$ such that $\left(E, H^{\prime}, F\right)$ is a standard triple. Since $H^{\prime}$ is a real semisimple element there exists $g \in G$ such that $g \cdot H^{\prime} \in \mathfrak{a}$. Hence, without any loss of generality, we may assume that $H^{\prime} \in \mathfrak{a}$. We can choose an element $w$ in the Weyl group of $\Sigma$ such that $w \cdot H^{\prime} \in C_{\Theta}$. This Weyl group can be identified with $N_{G}(\mathfrak{a}) / Z_{G}(\mathfrak{a})$. Hence we can replace $H^{\prime}$ with $w \cdot H^{\prime}$, i.e., we may assume that $H^{\prime} \in C_{\Theta}$ and consequently $H^{\prime}=H$. Then $E \in \mathcal{O}(2)$, and so the map mentioned in the theorem is surjective.

Next let $\mathcal{O}_{1}, \mathcal{O}_{2} \subset \mathcal{O}(2)$ be $Z_{G}(H)$-orbits and assume that $\mathcal{O}_{1}$ and $\mathcal{O}_{2}$ are contained in the same $G$-orbit. Let $E_{1} \in \mathcal{O}_{1}$ and $E_{2} \in \mathcal{O}_{2}$. By our 
assumption, $E_{2} \in G \cdot E_{1}$. We can choose $F_{1}, F_{2} \in \mathfrak{g}(-2)$ such that $\left(E_{1}, H, F_{1}\right)$ and $\left(E_{2}, H, F_{2}\right)$ are standard triples. By [2, Chapter 8, $\S 11$, Proposition 1], there exists $g \in G$ such that

$$
g \cdot\left(E_{1}, H, F_{1}\right)=\left(E_{2}, H, F_{2}\right) .
$$

Hence $g \in Z_{G}(H)$ and $E_{2}=g \cdot E_{1}$. Consequently $\mathcal{O}_{1}=\mathcal{O}_{2}$, and so our map is also injective.

This theorem reduces the classification problem for nilpotent $G$-orbits in $\mathfrak{g}$ to the problem of classifying the $Z_{G}(H)$-orbits in $\mathcal{O}(2)$ for each of the characteristics $H$ satisfying $\sigma(H)=H$. In spite of its attractiveness, this method is hard to apply in practise. We used it in order to check the (known) classification in the case where $\mathfrak{g}$ is the split real form of $G_{2}$.

The general problem of classifying the nilpotent $G$-orbits in $\mathfrak{g}$, reduces easily to the case where $\mathfrak{g}$ is absolutely simple, i.e., $\mathfrak{g}^{c}$ is simple. If $\mathfrak{g}$ is of classical type, the classification is well known [17]. When $\mathfrak{g}$ is a noncompact real form of an exceptional algebra $\mathfrak{g}^{c}$, the classification was obtained in our papers $[6,7]$. In fact we classified the nonzero nilpotent $K^{c}$-orbits in $\mathfrak{p}^{c}$. In view of Sekiguchi bijection, this provides indirectly also a classification of the nonzero nilpotent $G$-orbits in $\mathfrak{g}$.

Let us recall the following basic theorem of Kostant and Rallis [13].

Theorem 3. Every nonzero nilpotent element $E \in \mathfrak{p}^{c}$ is the nilpositive element of a normal triple. If $(E, H, F)$ and $\left(E^{\prime}, H^{\prime}, F^{\prime}\right)$ are normal triples such that $E=E^{\prime}$ or $H=H^{\prime}$, then there exists $g \in K^{c}$ such that $g \cdot(E, H, F)=\left(E^{\prime}, H^{\prime}, F^{\prime}\right)$.

Let $\mathfrak{t}$ be a Cartan subalgebra of $\mathfrak{k}, \Delta$ the root system of $\left(\mathfrak{k}^{c}, \mathfrak{t}^{c}\right)$ and $C_{\Delta} \subset i \mathfrak{t}$ the closed fundamental Weyl chamber corresponding to some base $\Gamma$ of $\Delta$. Let $\mathcal{O} \subset \mathfrak{p}^{c}$ be a nilpotent $K^{c}$-orbit. The above theorem implies that there exists a normal triple $(E, H, F)$ with $E \in \mathcal{O}$ and $H \in C_{\Delta}$. Furthermore the neutral element $H$ of this triple is uniquely determined by $\mathcal{O}$ and we refer to it as the characteristic of $\mathcal{O}$.

From now on we assume that $\mathfrak{g}^{c}$ is an exceptional simple complex Lie algebra and that $\mathfrak{g}$ is noncompact.

Assume first that $\mathfrak{k}$ is semisimple. Then the Dynkin diagram of $(\Delta, \Gamma)$ together with the labels $\alpha(H), \alpha \in \Gamma$, determines the characteristic $H$ uniquely. (The labels $\alpha(H)$ are nonnegative integers.)

Next assume that $\mathfrak{k}$ is not semisimple. Then $\mathfrak{k}=\mathfrak{k}_{0} \oplus \mathfrak{z}$ where $\mathfrak{k}_{0}$ is the derived subalgebra of $\mathfrak{k}$ and $\mathfrak{z}$ is the 1 -dimensional center of $\mathfrak{k}$. In this case the 
labels $\alpha(H), \alpha \in \Gamma$, determine only the component of $H$ in $\mathfrak{t}^{c} \cap \mathfrak{k}_{0}^{c}$, and say nothing about the component of $H$ in $\mathfrak{z}^{c}$. Since $\mathfrak{t}^{c}$ is also a Cartan subalgebra of $\mathfrak{g}^{c}$, the root system $\tilde{\Delta}$ of $\left(\mathfrak{g}^{c}, \mathfrak{t}^{c}\right)$ contains $\Delta$ as a closed root subsystem. Furthermore

$$
\operatorname{rank}(\tilde{\Delta})=\operatorname{rank}(\Delta)+1
$$

In this case we can choose a $\operatorname{root} \beta \in \tilde{\Delta}$ such that $\tilde{\Gamma}=\Gamma \cup\{\beta\}$ is a base of $\tilde{\Delta}([6])$. This $\beta$ is not unique: There are exactly two such choices. Once the choice of $\beta$ is made then the component of $H$ in $\mathfrak{z}^{c}$ is uniquely determined by the integer $\beta(H)$. Hence the labels $\alpha(H), \alpha \in \tilde{\Gamma}$, determine $H$ uniquely.

\section{Structure constants and the action of $\sigma$.}

In the next section we shall tabulate the representatives $(E, H, F)$ of $K$ orbits of the real Cayley triples in $\mathfrak{g}$ when $\mathfrak{g}^{c}$ is of type $G_{2}, F_{4}$, or $E_{6}$. The nilpositive elements $E$ of these triples are the representatives of the nonzero nilpotent $G$-orbits in $\mathfrak{g}$. In order to do this we have to choose a suitable basis of $\mathfrak{g}^{c}$ and describe the action of $\sigma$ in terms of that basis. This section is devoted to that task.

Let $n$ be the rank of $\mathfrak{g}^{c}$ and $N$ the number of positive roots of $(\Phi, \Pi)$. We start by enumerating the simple roots :

$$
\Pi=\left\{\alpha_{1}, \alpha_{2}, \ldots, \alpha_{n}\right\}
$$

in the same way as in [1]. A positive root

$$
\alpha=k_{1} \alpha_{1}+k_{2} \alpha_{2}+\cdots+k_{n} \alpha_{n}
$$

will be also represented by the symbol $k_{1} k_{2} \cdots k_{n}$. (Note that each $k_{i}$ is a single digit.) The height of $\alpha$ is defined by

$$
\operatorname{ht}(\alpha)=k_{1}+k_{2}+\cdots+k_{n}
$$

We extend the enumeration of simple roots to obtain an enumeration

$$
\alpha_{1}, \alpha_{2}, \ldots, \alpha_{n}, \ldots, \alpha_{N}
$$

of all positive roots such that ht $\left(\alpha_{i}\right) \leq \mathrm{ht}\left(\alpha_{j}\right)$ for $i<j$. In particular $\alpha_{N}$ is the highest root of $(\Phi, \Pi)$. The negative root $-\alpha_{i}$ will also be written as $\alpha_{-i}, 1 \leq i \leq N$.

The extended Dynkin diagrams of $G_{2}, F_{4}$, and $E_{6}$ are given in Fig. 1. 


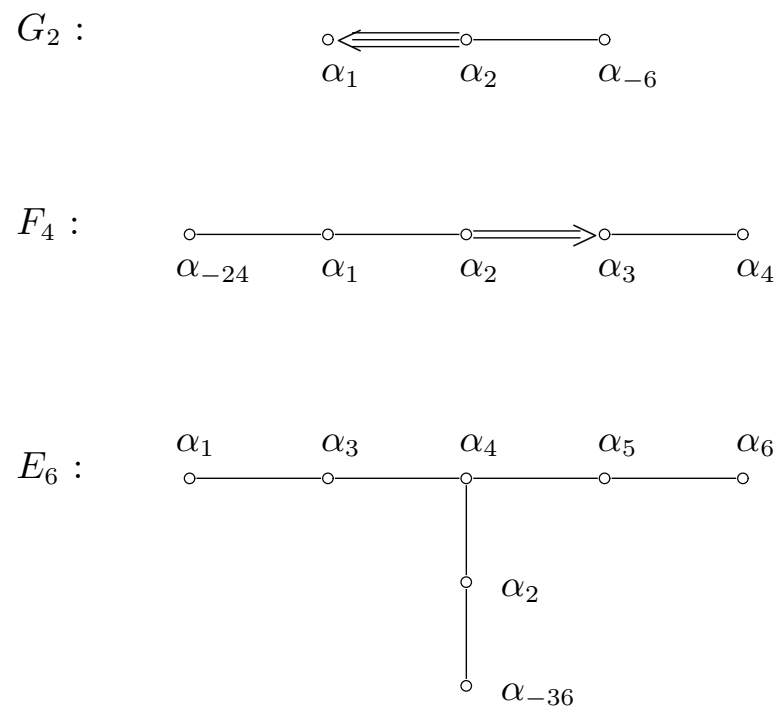

Figure 1.

The co-root $H_{\alpha_{i}}$ will be now denoted by $H_{i}$. Hence

$$
\Pi^{\vee}:=\left\{H_{1}, \ldots, H_{n}\right\}
$$

is a base of the dual root system $\Phi^{\vee}$ of $\Phi$. A positive co-root $H_{i}, i>0$, can be written as

$$
H_{i}=k_{1}^{\prime} H_{1}+\cdots+k_{n}^{\prime} H_{n}
$$

where $k_{i}^{\prime}$ are nonnegative integers. Thus we can represent $H_{i}$ by the symbol $k_{1}^{\prime} k_{2}^{\prime} \cdots k_{n}^{\prime}$.

If $\mathfrak{g}^{c}$ is simply laced, say of type $E_{6}$, then a positive root $\alpha_{i}$ and its co-root $H_{i}$ will be represented by the same symbol. This is not the case if $\mathfrak{g}^{c}$ is triply or doubly laced (say of type $G_{2}$ or $F_{4}$ ). 
In Tables 1-3 we list the positive roots $\alpha_{i}$ and co-roots $H_{i}$ for $G_{2}, F_{4}$, and $E_{6}$, respectively.

Table 1.

Positive roots of $G_{2}$.

\begin{tabular}{|ccc|ccc|ccc|}
\hline$i$ & $\alpha_{i}$ & $H_{i}$ & $i$ & $\alpha_{i}$ & $H_{i}$ & $i$ & $\alpha_{i}$ & $H_{i}$ \\
\hline 1 & 10 & 10 & 3 & 11 & 13 & 5 & 31 & 11 \\
2 & 01 & 01 & 4 & 21 & 23 & 6 & 32 & 12 \\
\hline
\end{tabular}

Table 2.

Positive roots of $F_{4}$.

\begin{tabular}{|rrr|rrr|rrr|}
\hline$i$ & $\alpha_{i}$ & $H_{i}$ & $i$ & $\alpha_{i}$ & $H_{i}$ & $i$ & $\alpha_{i}$ & $H_{i}$ \\
\hline 1 & 1000 & 1000 & 9 & 0120 & 0110 & 17 & 1221 & 2421 \\
2 & 0100 & 0100 & 10 & 0111 & 0211 & 18 & 1122 & 1111 \\
3 & 0010 & 0010 & 11 & 1120 & 1110 & 19 & 1231 & 2431 \\
4 & 0001 & 0001 & 12 & 1111 & 2211 & 20 & 1222 & 1211 \\
5 & 1100 & 1100 & 13 & 0121 & 0221 & 21 & 1232 & 2432 \\
6 & 0110 & 0210 & 14 & 1220 & 1210 & 22 & 1242 & 1221 \\
7 & 0011 & 0011 & 15 & 1121 & 2221 & 23 & 1342 & 1321 \\
8 & 1110 & 2210 & 16 & 0122 & 0111 & 24 & 2342 & 2321 \\
\hline
\end{tabular}

Table 3.

Positive roots of $E_{6}$.

\begin{tabular}{|rr|rr|rr|rr|}
\hline$i$ & $\alpha_{i}, H_{i}$ & $i$ & $\alpha_{i}, H_{i}$ & $i$ & $\alpha_{i}, H_{i}$ & $i$ & $\alpha_{i}, H_{i}$ \\
\hline 1 & 100000 & 10 & 000110 & 19 & 011110 & 28 & 011211 \\
2 & 010000 & 11 & 000011 & 20 & 010111 & 29 & 112210 \\
3 & 001000 & 12 & 101100 & 21 & 001111 & 30 & 111211 \\
4 & 000100 & 13 & 011100 & 22 & 111110 & 31 & 011221 \\
5 & 000010 & 14 & 010110 & 23 & 101111 & 32 & 112211 \\
6 & 000001 & 15 & 001110 & 24 & 011210 & 33 & 111221 \\
7 & 101000 & 16 & 000111 & 25 & 011111 & 34 & 112221 \\
8 & 010100 & 17 & 111100 & 26 & 111210 & 35 & 112321 \\
9 & 001100 & 18 & 101110 & 27 & 111111 & 36 & 122321 \\
\hline
\end{tabular}


If $\sigma\left(\alpha_{i}\right)=\alpha_{j}$, we shall also write $\sigma(i)=j$. In this way $\sigma$ defines an involutory permutation of the set

$$
\{ \pm 1, \pm 2, \ldots, \pm N\}
$$

In this notation, we have $\sigma\left(H_{i}\right)=H_{\sigma(i)}$ for all $i$. We can choose nonzero elements $X_{i} \in \mathfrak{g}_{i}^{c}$, where $\mathfrak{g}_{i}^{c}:=\mathfrak{g}_{\alpha_{i}}^{c}$ is the root space of $\alpha_{i}$, such that (see [2]):

(i) $\left[X_{-i}, X_{i}\right]=H_{i}$ for all $i$;

(ii) the linear map $\omega: \mathfrak{g}^{c} \rightarrow \mathfrak{g}^{c}$ which is -1 on $\mathfrak{h}^{c}$ and satisfies $\omega\left(X_{i}\right)=X_{-i}$ for all $i$ is an automorphism of $\mathfrak{g}^{c}$.

If $i+j=0$, then the bracket $\left[X_{i}, X_{j}\right]$ is determined by (i). Assume now that $i+j \neq 0$. If $\alpha_{i}+\alpha_{j}$ is not a root then $\left[X_{i}, X_{j}\right]=0$ and we set $N(i, j)=0$. If $\alpha_{i}+\alpha_{j}=\alpha_{k} \in \Phi$, then we define $N(i, j)$ by

$$
\left[X_{i}, X_{j}\right]=N(i, j) X_{k}
$$

The conditions (i) and (ii) imply that $N(i, j)$ is an integer. More precisely, let $p, q \geq 0$ be the largest integers such that

$$
\alpha_{j}+p \alpha_{i}, \alpha_{j}-q \alpha_{i} \in \Phi
$$

Assuming that $\alpha_{i}+\alpha_{j}=\alpha_{k} \in \Phi$, i.e., $p \geq 1$, we have

$$
N(i, j)=\varepsilon(i, j)(q+1)
$$

where $\varepsilon(i, j)= \pm 1$.

The $X_{i}$ 's together with the $H_{1}, \ldots, H_{n}$ form a basis of $\mathfrak{g}^{c}$. All the brackets of these basic elements are uniquely determined except for the signs $\varepsilon(i, j)$.

The conditions (i) and (ii) do not determine the $X_{i}$ 's uniquely. Different choices of the $X_{i}$ 's may produce different signs $\varepsilon(i, j)$. As shown by J. Kurtzke [14], the $X_{i}$ 's can be chosen, not only to satisfy (i) and (ii), but also (iii) $\varepsilon(i, j)=1$ for $1 \leq i \leq n<j \leq N$;

(iv) $\varepsilon(i, j)=-\varepsilon(j, k)$ whenever $\alpha_{i}, \alpha_{j}, \alpha_{k}$ are consecutively linked simple roots in the Dynkin diagram.

For $G_{2}$ and $F_{4}$ we specify that $\varepsilon(1,2)=1$, and for $E_{6}$ we specify that $\varepsilon(1,3)=1$. All other $\varepsilon(i, j)$ are then uniquely determined by (iii) and (iv) (see [14]). In the Appendix we give the tables of the signs $\varepsilon(i, j)$, $1 \leq i, j \leq N$, for the complex simple Lie algebras $G_{2}, F_{4}$, and $E_{6}$.

If $Y \in \mathfrak{h}^{c}$ and we replace the $X_{i}$ 's with $\exp (\operatorname{ad} Y)\left(X_{i}\right)$, then (i-iv) remain valid. In fact the structure constants $N(i, j)$ do not change at all. One can choose such $Y$ so that the new $X_{i}$ 's satisfy also the condition

(v) $\sigma\left(X_{i}\right)=\xi_{i} X_{\sigma(i)}, \xi_{i}= \pm 1$, for all $i$. 
For details, see [15]. Furthermore one knows that $\xi_{i}=1$ for all $\alpha_{i} \in \Phi_{0}$, and $\xi_{-i}=\xi_{i}$ for all $i$. If $\alpha_{i}+\alpha_{j}=\alpha_{k}$, then

$$
\xi_{k} N(i, j)=\xi_{i} \xi_{j} N(\sigma(i), \sigma(j))
$$

holds. It follows that all the $\xi_{i}$ 's can be computed provided that $\xi_{1}, \ldots, \xi_{n}$ are known.

For all non-compact real forms of $G_{2}, F_{4}$, and $E_{6}$ we may choose $\xi_{i}=1$ for $1 \leq i \leq n$, with one exception: For the real form $E_{6(-14)}$ of $E_{6}$ we can set $\xi_{i}=1$ for $1 \leq i \leq 5$, while $\xi_{6}=-1$.

In order to pin down the action of $\sigma$ on the $X_{i}$ 's, we still need to determine the corresponding permutation of the set (4.1). This permutation is determined by the Satake diagram of $\mathfrak{g}$ (see [15]). For readers convenience we shall describe this permutation below.

If $\mathfrak{g}$ is split, then $\sigma$ is the identity permutation. Assume now that $\mathfrak{g}$ is not split. Since $\sigma$ induces an automorphism of $\Phi$, it suffices to specify $\sigma(i)$ for $1 \leq i \leq n$. If the vertex $\alpha_{i}$ in the Satake diagram of $\mathfrak{g}$ is black, then we have $\sigma(i)=-i$. For the white vertices of the Satake diagram, the action of $\sigma$ is given as follows:

$$
\begin{array}{ll}
F_{4(-20)}: & \sigma(4)=19 \\
E_{6(2)}: & \sigma(1)=6, \sigma(2)=2, \sigma(3)=5, \sigma(4)=4, \sigma(5)=3, \sigma(6)=1 ; \\
E_{6(-14)}: & \sigma(1)=21, \sigma(2)=24, \sigma(6)=18 \\
E_{6(-26)}: & \sigma(1)=29, \sigma(6)=31
\end{array}
$$

\section{Explicit Cayley triples.}

In Tables 4-10 we list the representatives $(E, H, F)$ of the $G$-orbits of real Cayley triples in $\mathfrak{g}$. Here $\mathfrak{g}$ is one of the non-compact real forms of $G_{2}, F_{4}$, or $E_{6}$. Thus $\mathfrak{g}$ is one of the algebras:

$$
G_{2(2)}, F_{4(4)}, F_{4(-20)}, E_{6(6)}, E_{6(2)}, E_{6(-14)}, E_{6(-26)},
$$

where the number in parentheses is the Cartan index of $\mathfrak{g}$, i.e, the difference $\operatorname{dim} \mathfrak{p}-\operatorname{dim} \mathfrak{k}$.

The neutral element $H$ is always chosen to be the characteristic of the nonzero nilpotent orbit $G^{c} \cdot E$. By Theorem 1 we know that $H \in \mathfrak{a}$. We list both: The labels $\alpha_{i}(H), i=1, \ldots, n$, and the coefficients $k_{1}, \ldots, k_{n}$ in

$$
H=k_{1} H_{1}+\cdots+k_{n} H_{n} .
$$

We tabulate only the neutral elements $H$ and the nilpositive elements $E$. The elements $F$ can easily be computed because $\theta=\sigma_{u} \sigma$ and $\sigma(E)=E$. 
So, we have

$$
F=\theta(E)=\sigma_{u} \sigma(E)=\sigma_{u}(E)
$$

We recall that $\sigma_{u}\left(X_{i}\right)=X_{-i}$ for all $i$.

In order to avoid possible confusion, we have used the same numbering of the nonzero nilpotent orbits as in our papers [6, 7]. That numbering was also used in [4] where the trivial orbit is appropriately given the number 0 .

Let us now sketch the method used to construct the Tables 4-10. By Theorem 2 we know the characteristics $H$ of the required representatives of nonzero nilpotent $G$-orbits, or equivalently the neutral elements of the representatives of $K$-orbits of real Cayley triples. Given such $H$, we denote by $I$ the set of indices $i$ such that $\left[H, X_{i}\right]=2 X_{i}$. Then $i>0$ for each $i \in I$ and the $X_{i}$ 's for $i \in I$ form a basis of the subspace $\mathfrak{g}(2)^{c}$ (see Section 3). For each subset $J \subset I$ let $\mathfrak{g}(2, J)^{c}$ be the (complex) subspace of $\mathfrak{g}(2)^{c}$ spanned by the $X_{i}$ 's with $i \in J$.

We search for all subsets $J \subset I$ satisfying the following three conditions:

(i) $\mathfrak{g}(2, J)^{c}$ is stable under $\sigma$;

(ii) if $i, j \in J$, then $\alpha_{i}-\alpha_{j}$ is not a root;

(iii) $H$ belongs to the complex subspace spanned by all $H_{i}$ with $i \in J$.

(More precisely, we only need to find the representatives of the orbits of the Weyl group of $\Phi_{0}$ acting on the collection of all subsets $J \subset I$ satisfying the above three conditions.)

Given such $J$ we set

$$
E=\Sigma_{i \in J} c_{i} X_{i}
$$

where $c_{i}$ are nonzero scalars chosen so that $\sigma(E)=E$, i.e., $E \in \mathfrak{g}$. For each such $E$, there exists $F \in \mathfrak{g}(-2)=\mathfrak{g} \cap \mathfrak{g}(-2)^{c}$ such that $[F, E]=H$. If there are no subsets $J \subset I$ having properties (i-iii), then one has to construct a suitable $E \in \mathfrak{g}(2)$ by a more elaborate procedure. In most cases the required sets $J$ exist.

After constructing several such Cayley triples $(E, H, F)$, we are faced with the problem of identifying the orbits to which they belong. For that purpose we have to pass to their Cayley transforms $\left(E^{\prime}, H^{\prime}, F^{\prime}\right)$. In fact we only need the neutral elements $H^{\prime}=i(E+F)$.

Let $\mathcal{O}^{c}=G^{c} \cdot E$. If $\mathcal{O}^{c} \cap \mathfrak{g}$ is a single $G$-orbit, then the identification problem mentioned above is trivial. Otherwise $\mathcal{O}^{c} \cap \mathfrak{g}$ is a union of two or three $G$-orbits. In most cases the invariant

$$
\operatorname{inv}:=\operatorname{dim} Z_{\mathfrak{k} c}\left(H^{\prime}\right)
$$


distinguishes these $G$-orbits. This invariant is listed in the last column of our Tables 4-10. If this crude invariant fails to distinguish between the various $G$ orbits in $\mathcal{O}^{c} \cap \mathfrak{g}$, we use the spectrum of the linear operator ad $\left(H^{\prime}\right)$ restricted to $\mathfrak{k}^{c}$.

When $\mathfrak{g}$ is of type $E_{6(2)}$ or $E_{6(-14)}$, there exist pairs of $G$-orbits, say $\mathcal{O}^{\prime}$ and $\mathcal{O}^{\prime \prime}$ contained in the same nilpotent $G$-orbit $\mathcal{O}^{c}$ which cannot be distinguished even by this finer invariant. In these cases we have $\mathcal{O}^{\prime \prime}=-\mathcal{O}^{\prime}$ and there exists an outer automorphism of $\mathfrak{g}$ which interchanges $\mathcal{O}^{\prime}$ and $\mathcal{O}^{\prime \prime}$ (see [8]). Such pairs $\left\{\mathcal{O}^{\prime}, \mathcal{O}^{\prime \prime}\right\}$ are recorded jointly on the same line in Tables 8 and 9 , and the nilpositive element is written as $\pm E$. The two sign choices give two different orbits. This ambiguity is caused by the fact that, in the two cases mentioned above, the automorphism group of the Dynkin diagram of $\left(\mathfrak{k}^{c}, \mathfrak{t}^{c}\right)$ has order 2. In the other cases, this automorphism group is trivial.

The number $\zeta$ that occurs in Table 8 (orbits 12 and 13) is a primitive cube root of 1 .

By using the Tables 1-3 and those in the Appendix, one can verify that our Tables 4-10 indeed give real Cayley triples. We give full details for the orbit 36 of Table 8 , where $\mathfrak{g}$ is of the type $E_{6(2)}$.

In that case we have

$$
E=4 X_{2}+\sqrt{12}\left(X_{1}+X_{6}\right)+\sqrt{7}\left(X_{3}+X_{5}\right)+i \sqrt{15}\left(X_{9}-X_{10}\right) .
$$

As $\alpha_{3}+\alpha_{4}=\alpha_{9}$, it follows from (4.3) that

$$
\xi_{9} \varepsilon(3,4)=\xi_{3} \xi_{4} \varepsilon(\sigma(3), \sigma(4))=\varepsilon(5,4) .
$$

By consulting Table 13 , we find that $\varepsilon(3,4)=\varepsilon(5,4)=-1$, and so $\xi_{9}=1$. As

$$
\sigma\left(X_{1}\right)=X_{6}, \sigma\left(X_{2}\right)=X_{2}, \sigma\left(X_{3}\right)=X_{5}
$$

and

$$
\sigma\left(X_{9}\right)=\xi_{9} X_{\sigma(9)}=X_{10},
$$

we conclude that $\sigma(E)=E$, i.e., $E \in \mathfrak{g}$. Hence (5.2) gives

$$
F=4 X_{-2}+\sqrt{12}\left(X_{-1}+X_{-6}\right)+\sqrt{7}\left(X_{-3}+X_{-5}\right)-i \sqrt{15}\left(X_{-9}-X_{-10}\right) .
$$

Clearly we have $F \in \mathfrak{g}$. It remains to verify that $[F, E]=H$, where

$$
H=12\left(H_{1}+H_{6}\right)+22\left(H_{3}+H_{5}\right)+16 H_{2}+30 H_{4} .
$$


A direct computation gives

$$
\begin{aligned}
{[F, E]=} & 16\left[X_{-2}, X_{2}\right]+12\left[X_{-1}, X_{1}\right]+12\left[X_{-6}, X_{6}\right] \\
& +7\left[X_{-3}, X_{3}\right]+7\left[X_{-5}, X_{5}\right]+15\left[X_{-9}, X_{9}\right]+15\left[X_{-10}, X_{10}\right] \\
& +i \sqrt{105}\left(\left[X_{-3}, X_{9}\right]-\left[X_{-5}, X_{10}\right]-\left[X_{-9}, X_{3}\right]+\left[X_{-10}, X_{5}\right]\right) \\
= & 16 H_{2}+12\left(H_{1}+H_{6}\right)+7\left(H_{3}+H_{5}\right)+15\left(H_{9}+H_{10}\right) \\
& +i \sqrt{105}\left[(N(-3,9)-N(-5,10)) X_{4}+(N(-10,5)-N(-9,3)) X_{-4}\right] .
\end{aligned}
$$

To compute these structure constants, we use the fact that $\varepsilon(i, j)=-\varepsilon(-i, k)$ if $\alpha_{i}+\alpha_{j}=\alpha_{k}$, see [2, Chapter VIII, $\S 2$, Lemma 4]. Hence $\varepsilon(-3,9)=$ $-\varepsilon(3,4)=1$ and $\varepsilon(-5,10)=-\varepsilon(3,4)=1$ (see Tables 3 and 13). We now apply (4.2), using Table 3 in order to compute the integer $q$, and obtain that $N(-3,9)=N(-5,10)=1$. Since $N(\beta, \alpha)=-N(\alpha, \beta)$ and $N(-\alpha,-\beta)=N(\alpha, \beta)$, see [2, Chapter VIII, $\S 2$, Proposition 7$], N(-9,3)=$ $N(-10,5)=-1$. Since $H_{9}=H_{3}+H_{4}, H_{10}=H_{4}+H_{5}$ (see Table 3), we deduce that indeed $[F, E]=H$.

In this example the set $I$ is $\{1,2,3,5,6,8,9,10\}$ and there is no subset $J \subset I$ satisfying all three conditions (i-iii). We used the subset $J=$ $\{1,2,3,5,6,9,10\}$ which satisfies the conditions (i) and (iii), but not (ii).

Table 4.

Cayley triples in $G_{2(2)}$.

\begin{tabular}{|c|c|c|c|r|}
\hline & $\alpha_{i}(H)$ & $k_{i}$ & $E$ & inv \\
\hline 1 & 01 & 1,2 & $X_{6}$ & 2 \\
2 & 10 & 2,3 & $X_{4}$ & 2 \\
3 & 02 & 2,4 & $X_{2}+X_{4}$ & 2 \\
4 & 02 & 2,4 & $X_{2}-X_{4}$ & 4 \\
5 & 22 & 6,10 & $X_{1}+X_{2}$ & 2 \\
\hline
\end{tabular}


Table 5.

Cayley triples in $F_{4(4)}$.

\begin{tabular}{|c|c|c|c|c|}
\hline & $\alpha_{i}(H)$ & $k_{i}$ & $E$ & inv \\
\hline 1 & 1000 & $2,3,2,1$ & $X_{24}$ & 10 \\
\hline 2 & 0001 & $2,4,3,2$ & $X_{16}+X_{24}$ & 12 \\
\hline 3 & 0001 & $2,4,3,2$ & $X_{21}$ & 10 \\
\hline 4 & 0100 & $3,6,4,2$ & $X_{14}+X_{20}+X_{22}$ & 10 \\
\hline 5 & 0100 & $3,6,4,2$ & $X_{14}+X_{21}$ & 6 \\
\hline 6 & 2000 & $4,6,4,2$ & $X_{1}+X_{14}+X_{20}+X_{22}$ & 22 \\
\hline 7 & 2000 & $4,6,4,2$ & $X_{8}+X_{18}+X_{23}$ & 14 \\
\hline 8 & 2000 & $4,6,4,2$ & $\sqrt{2}\left(X_{1}+X_{23}\right)$ & 10 \\
\hline 9 & 0002 & $4,8,6,4$ & $\sqrt{2}\left(X_{4}+X_{19}\right)$ & 10 \\
\hline 10 & 0010 & $4,8,6,3$ & $X_{15}+\sqrt{2}\left(X_{9}+X_{20}\right)$ & 6 \\
\hline 11 & 2001 & $6,10,7,4$ & $\sqrt{3}\left(X_{1}+X_{14}\right)+2 X_{16}$ & 6 \\
\hline 12 & 2001 & $6,10,7,4$ & $\sqrt{3} X_{8}+2 X_{16}$ & 6 \\
\hline 13 & 0101 & $5,10,7,4$ & $\sqrt{2}\left(X_{10}+X_{15}\right)+X_{14}$ & 4 \\
\hline 14 & 1010 & $6,11,8,4$ & $\sqrt{3} X_{8}+X_{9}-2 X_{16}$ & 6 \\
\hline 15 & 1010 & $6,11,8,4$ & $\sqrt{3} X_{8}+X_{9}+2 X_{16}$ & 4 \\
\hline
\end{tabular}


DRAGOMIR Ž. DOKOVIĆ

Table 5.

(continued)

\begin{tabular}{|c|c|c|c|c|}
\hline & $\alpha_{i}(H)$ & $k_{i}$ & $E$ & inv \\
\hline 16 & 0200 & $6,12,8,4$ & $X_{2}+\sqrt{3} X_{8}+X_{9}-2 X_{16}$ & 12 \\
17 & 0200 & $6,12,8,4$ & $X_{2}+\sqrt{3} X_{8}+X_{9}+2 X_{16}$ & 8 \\
18 & 0200 & $6,12,8,4$ & $X_{2}+\sqrt{3} X_{8}-X_{9}+2 X_{16}$ & 6 \\
19 & 2200 & $10,18,12,6$ & $\sqrt{10} X_{1}+\sqrt{6}\left(X_{2}+X_{9}+X_{16}\right)$ & 10 \\
20 & 2200 & $10,18,12,6$ & $\sqrt{10} X_{1}+\sqrt{6}\left(X_{2}+X_{13}\right)$ & 6 \\
21 & 1012 & $10,19,14,8$ & $2 \sqrt{2} X_{4}+\sqrt{5} X_{8}+3 X_{9}$ & 4 \\
22 & 0202 & $10,20,14,8$ & $X_{2}+2 \sqrt{2} X_{4}+\sqrt{5} X_{8}+3 X_{9}$ & 8 \\
23 & 0202 & $10,20,14,8$ & $X_{2}+2 \sqrt{2} X_{4}+\sqrt{5} X_{8}-3 X_{9}$ & 4 \\
24 & 2202 & $14,26,18,10$ & $\sqrt{14} X_{1}+2 \sqrt{2} X_{2}+\sqrt{10} X_{4}-3 \sqrt{2} X_{9}$ & 4 \\
25 & 2202 & $14,26,18,10$ & $\sqrt{14} X_{1}+2 \sqrt{2} X_{2}+\sqrt{10} X_{4}+3 \sqrt{2} X_{9}$ & 6 \\
26 & 2222 & $22,42,30,16$ & $\sqrt{22} X_{1}+\sqrt{42} X_{2}+\sqrt{30} X_{3}+4 X_{4}$ & 4 \\
\hline
\end{tabular}

Table 6.

Cayley triples in $F_{4(-20)}$.

\begin{tabular}{|c|c|c|c|c|}
\hline & $\alpha_{i}(H)$ & $k_{i}$ & $E$ & inv \\
\hline 1 & 0001 & $2,4,3,2$ & $X_{21}$ & 16 \\
2 & 0002 & $4,8,6,4$ & $\sqrt{2}\left(X_{4}+X_{19}\right)$ & 22 \\
\hline
\end{tabular}


Table 7.

Cayley triples in $E_{6(6)}$.

\begin{tabular}{|c|c|c|c|c|}
\hline & $\alpha_{i}(H)$ & $k_{i}$ & $E$ & inv \\
\hline 1 & 010000 & $1,2,2,3,2,1$ & $X_{36}$ & 18 \\
\hline 2 & 100001 & $2,2,3,4,3,2$ & $X_{23}+X_{36}$ & 16 \\
\hline 3 & 000100 & $2,3,4,6,4,2$ & $X_{24}+X_{30}+X_{34}$ & 12 \\
\hline 4 & 020000 & $2,4,4,6,4,2$ & $\sqrt{2}\left(X_{2}+X_{35}\right)$ & 18 \\
\hline 5 & 020000 & $2,4,4,6,4,2$ & $X_{2}+X_{24}+X_{30}-X_{34}$ & 24 \\
\hline 6 & 200002 & $4,4,6,8,6,4$ & $\sqrt{2}\left(X_{1}+X_{6}+X_{29}+X_{31}\right)$ & 16 \\
\hline 7 & 120001 & $4,6,7,10,7,4$ & $\sqrt{3}\left(X_{2}+X_{24}\right)+2 X_{23}$ & 10 \\
\hline 8 & 110001 & $3,4,5,7,5,3$ & $\sqrt{2}\left(X_{17}+X_{31}\right)+X_{23}$ & 10 \\
\hline 9 & 220002 & $6,8,10,14,10,6$ & $\sqrt{6}\left(X_{1}+X_{21}\right)+2\left(X_{2}+X_{24}\right)$ & 10 \\
\hline 10 & 001010 & $3,4,6,8,6,3$ & $X_{15}+X_{23}+\sqrt{2}\left(X_{22}+X_{28}\right)$ & 10 \\
\hline 11 & 100101 & $4,5,7,10,7,4$ & $X_{24}+\sqrt{2}\left(X_{12}+X_{16}+X_{22}+X_{25}\right)$ & 8 \\
\hline 12 & 000200 & $4,6,8,12,8,4$ & $X_{4}+X_{15}+2 X_{23}+\sqrt{3}\left(X_{13}+X_{14}\right)$ & 12 \\
\hline 13 & 020200 & $6,10,12,18,12,6$ & $\sqrt{10} X_{2}+\sqrt{6}\left(X_{4}+X_{15}+X_{23}\right)$ & 12 \\
\hline 14 & 211012 & $8,10,14,19,14,8$ & $\begin{array}{c}2 \sqrt{2}\left(X_{1}+X_{6}\right)+\sqrt{5}\left(X_{13}+X_{14}\right) \\
+3 X_{15}\end{array}$ & 6 \\
\hline
\end{tabular}


DRAGOMIR Ž. DOKOVIĆ

Table 7.

(continued)

\begin{tabular}{|c|c|c|c|c|}
\hline & $\alpha_{i}(H)$ & $k_{i}$ & $E$ & inv \\
\hline 15 & 011010 & $4,6,8,11,8,4$ & $X_{15}+2 X_{23}+\sqrt{3}\left(X_{13}+X_{14}\right)$ & 8 \\
\hline 16 & 111011 & $6,8,11,15,11,6$ & $\sqrt{6}\left(X_{7}+X_{16}\right)+X_{15}+2\left(X_{13}+X_{14}\right)$ & 6 \\
\hline 17 & 121011 & $7,10,13,18,13,7$ & $\begin{array}{l}\frac{1}{\sqrt{7}}\left(2 \sqrt{15} X_{2}+2 \sqrt{6} X_{7}+\sqrt{10} X_{8}\right. \\
\left.\quad-5 X_{12}+\sqrt{42} X_{15}+7 X_{16}\right)\end{array}$ & 6 \\
\hline 18 & 222022 & $12,16,22,30,22,12$ & $\begin{array}{l}\sqrt{12}\left(X_{1}+X_{6}\right)+\sqrt{22} X_{5} \\
+\frac{1}{\sqrt{11}}\left(2 \sqrt{14} X_{2}+4 \sqrt{2} X_{3}\right. \\
\left.+2 \sqrt{30} X_{8}-\sqrt{210} X_{9}\right)\end{array}$ & 6 \\
\hline 19 & 200202 & $8,10,14,20,14,8$ & $\begin{aligned} X_{4}+ & 3 X_{15}+2 \sqrt{2}\left(X_{1}+X_{6}\right) \\
& +\sqrt{5}\left(X_{13}+X_{14}\right)\end{aligned}$ & 8 \\
\hline 20 & 222222 & $16,22,30,42,30,16$ & $\begin{array}{c}4\left(X_{1}+X_{6}\right)+\sqrt{30}\left(X_{3}+X_{5}\right) \\
\quad+\sqrt{22} X_{2}+\sqrt{42} X_{4}\end{array}$ & 6 \\
\hline 21 & 220202 & $10,14,18,26,18,10$ & $\begin{array}{l}\sqrt{10}\left(X_{1}+X_{6}\right)+\sqrt{14} X_{2} \\
\quad+\sqrt{2}\left(2 X_{4}+3 X_{15}\right)\end{array}$ & 8 \\
\hline 22 & 200202 & $8,10,14,20,14,8$ & $\begin{aligned} X_{4}- & 3 X_{15}+2 \sqrt{2}\left(X_{1}+X_{6}\right) \\
& +\sqrt{5}\left(X_{13}+X_{14}\right)\end{aligned}$ & 10 \\
\hline 23 & 000200 & $4,6,8,12,8,4$ & $X_{4}-X_{15}+2 X_{23}+\sqrt{3}\left(X_{13}+X_{14}\right)$ & 14 \\
\hline
\end{tabular}


Table 8.

Cayley triples in $E_{6(2)}$.

\begin{tabular}{|c|c|c|c|c|}
\hline & $\alpha_{i}(H)$ & $k_{i}$ & $E$ & inv \\
\hline 1 & 010000 & $1,2,2,3,2,1$ & $X_{36}$ & 18 \\
\hline 2 & 100001 & $2,2,3,4,3,2$ & $X_{23}+X_{36}$ & 18 \\
\hline 3 & 100001 & $2,2,3,4,3,2$ & $X_{32}+X_{33}$ & 14 \\
\hline 4 & 000100 & $2,3,4,6,4,2$ & $X_{24}+X_{30}+X_{34}$ & 18 \\
\hline 5 & 000100 & $2,3,4,6,4,2$ & $X_{24}+X_{30}-X_{34}$ & 10 \\
\hline 6 & 020000 & $2,4,4,6,4,2$ & $-X_{2}+X_{24}+X_{30}+X_{34}$ & 36 \\
\hline 7 & 020000 & $2,4,4,6,4,2$ & $X_{2}+X_{24}+X_{30}-X_{34}$ & 20 \\
\hline 8 & 020000 & $2,4,4,6,4,2$ & $\sqrt{2}\left(X_{2}+X_{35}\right)$ & 18 \\
\hline 9,10 & 110001 & $3,4,5,7,5,3$ & $\pm\left[X_{22}+X_{23}+X_{25}+i\left(X_{26}-X_{28}\right)\right]$ & 12 \\
\hline 11 & 200002 & $4,4,6,8,6,4$ & $\sqrt{2}\left(X_{1}+X_{6}+X_{29}+X_{31}\right)$ & 14 \\
\hline 12,13 & 001010 & $3,4,6,8,6,3$ & $\begin{array}{c} \pm \frac{1}{\sqrt{3}}\left[X_{15}+X_{19}-X_{23}+X_{24}-X_{27}\right. \\
-X_{30}+\sqrt{2}\left(X_{18}+X_{21}+\zeta X_{22}\right. \\
\left.\left.+\bar{\zeta} X_{25}+\bar{\zeta} X_{26}+\zeta X_{28}\right)\right]\end{array}$ & 16 \\
\hline 14 & 001010 & $3,4,6,8,6,3$ & $\sqrt{2}\left(X_{15}+X_{30}\right)+X_{22}+X_{25}$ & 8 \\
\hline 15 & 120001 & $4,6,7,10,7,4$ & $\sqrt{3}\left(X_{13}+X_{14}\right)-X_{15}+2 X_{23}$ & 10 \\
\hline 16 & 120001 & $4,6,7,10,7,4$ & $\sqrt{3}\left(X_{13}+X_{14}\right)+X_{15}+2 X_{23}$ & 10 \\
\hline
\end{tabular}


Table 8.

(continued)

\begin{tabular}{|c|c|c|c|c|}
\hline & $\alpha_{i}(H)$ & $k_{i}$ & $E$ & inv \\
\hline 17 & 100101 & $4,5,7,10,7,4$ & $\sqrt{2}\left(X_{12}+X_{16}+X_{22}+X_{25}\right)+X_{24}$ & 6 \\
\hline 18 & 011010 & $4,6,8,11,8,4$ & $\sqrt{3}\left(X_{13}+X_{14}\right)-X_{15}+2 X_{23}$ & 10 \\
\hline 19 & 011010 & $4,6,8,11,8,4$ & $\sqrt{3}\left(X_{13}+X_{14}\right)+X_{15}+2 X_{23}$ & 6 \\
\hline 20 & 000200 & $4,6,8,12,8,4$ & $X_{4}+\sqrt{3}\left(X_{13}+X_{14}\right)-X_{15}+2 X_{23}$ & 20 \\
\hline 21 & 000200 & $4,6,8,12,8,4$ & $-X_{4}+\sqrt{3}\left(X_{13}+X_{14}\right)+X_{15}+2 X_{23}$ & 12 \\
\hline 22 & 000200 & $4,6,8,12,8,4$ & $X_{4}+\sqrt{3}\left(X_{13}+X_{14}\right)+X_{15}+2 X_{23}$ & 10 \\
\hline 23 & 020200 & $6,10,12,18,12,6$ & $\sqrt{10} X_{2}+\sqrt{6}\left(-X_{4}+X_{15}+X_{23}\right)$ & 18 \\
\hline 24 & 020200 & $6,10,12,18,12,6$ & $\sqrt{10} X_{2}+\sqrt{6}\left(X_{4}+X_{15}+X_{23}\right)$ & 10 \\
\hline 25 & 220002 & $6,8,10,14,10,6$ & $\begin{array}{l}\sqrt{3}\left(i X_{1}-i X_{6}+X_{18}+X_{21}\right) \\
+\sqrt{2}\left(X_{2}+X_{8}+X_{19}-X_{24}\right)\end{array}$ & 18 \\
\hline 26 & 220002 & $6,8,10,14,10,6$ & $\begin{array}{c}\sqrt{3}\left(i X_{7}-i X_{11}+X_{12}+X_{16}\right) \\
\quad+2\left(X_{13}+X_{14}\right)\end{array}$ & 10 \\
\hline 27,28 & 111011 & $6,8,11,15,11,6$ & $\begin{array}{c} \pm\left[\sqrt{3}\left(X_{7}+X_{11}\right)+i \sqrt{3}\left(X_{12}-X_{16}\right)\right. \\
\left.2\left(X_{13}+X_{14}\right)+X_{15}\right]\end{array}$ & 6 \\
\hline 29,30 & 121011 & $7,10,13,18,13,7$ & $\begin{array}{l} \pm\left[X_{7}+X_{11}+i \sqrt{6}\left(X_{12}-X_{16}\right)\right. \\
\left.\quad+\sqrt{10} X_{2}+\sqrt{6} X_{15}\right]\end{array}$ & 8 \\
\hline 31 & 211012 & $8,10,14,19,14,8$ & $\begin{array}{l}3 X_{15}+2 \sqrt{2}\left(X_{1}+X_{6}\right) \\
\quad+\sqrt{5}\left(X_{13}+X_{14}\right)\end{array}$ & 6 \\
\hline
\end{tabular}


Table 8.

(continued)

\begin{tabular}{|c|c|c|c|c|}
\hline & $\alpha_{i}(H)$ & $k_{i}$ & $E$ & inv \\
\hline 32 & 200202 & $8,10,14,20,14,8$ & $\begin{aligned} X_{4}+ & 3 X_{15}+2 \sqrt{2}\left(X_{1}+X_{6}\right) \\
& +\sqrt{5}\left(X_{13}+X_{14}\right)\end{aligned}$ & 6 \\
\hline 33 & 200202 & $8,10,14,20,14,8$ & $\begin{aligned}-X_{4}+ & 3 X_{15}+2 \sqrt{2}\left(X_{1}+X_{6}\right) \\
& +\sqrt{5}\left(X_{13}+X_{14}\right)\end{aligned}$ & 12 \\
\hline 34 & 220202 & $10,14,18,26,18,10$ & $\begin{array}{l}\sqrt{10}\left(X_{1}+X_{6}\right)+\sqrt{14} X_{2} \\
\quad+\sqrt{2}\left(2 X_{4}+3 X_{15}\right)\end{array}$ & 6 \\
\hline 35 & 220202 & $10,14,18,26,18,10$ & $\begin{array}{l}\sqrt{10}\left(X_{1}+X_{6}\right)+\sqrt{14} X_{2} \\
\quad+\sqrt{2}\left(2 X_{4}-3 X_{15}\right)\end{array}$ & 10 \\
\hline 36 & 222022 & $12,16,22,30,22,12$ & $\begin{array}{c}4 X_{2}+\sqrt{12}\left(X_{1}+X_{6}\right) \\
+\sqrt{7}\left(X_{3}+X_{5}\right)+i \sqrt{15}\left(X_{9}-X_{10}\right)\end{array}$ & 8 \\
\hline 37 & 222222 & $16,22,30,42,30,16$ & $\begin{array}{c}4\left(X_{1}+X_{6}\right)+\sqrt{30}\left(X_{3}+X_{5}\right) \\
+\sqrt{22} X_{2}+\sqrt{42} X_{4}\end{array}$ & 6 \\
\hline
\end{tabular}


Table 9.

Cayley triples in $E_{6(-14)}$.

\begin{tabular}{|r|c|c|c|c|}
\hline & $\alpha_{i}(H)$ & $k_{i}$ & $E$ & inv \\
\hline 1,2 & 010000 & $1,2,2,3,2,1$ & $\pm i X_{36}$ & 26 \\
3,4 & 100001 & $2,2,3,4,3,2$ & $\pm i\left(X_{23}+X_{36}\right)$ & 30 \\
5 & 100001 & $2,2,3,4,3,2$ & $X_{27}-X_{35}$ & 18 \\
6 & 020000 & $2,4,4,6,4,2$ & $\sqrt{2}\left(X_{17}-X_{31}\right)$ & 20 \\
7,8 & 110001 & $3,4,5,7,5,3$ & $\pm\left[\sqrt{2}\left(X_{17}-X_{31}\right)+i X_{23}\right]$ & 12 \\
9 & 200002 & $4,4,6,8,6,4$ & $\sqrt{2}\left(X_{1}+X_{20}+X_{21}+X_{29}\right)$ & 30 \\
10,11 & 120001 & $4,6,7,10,7,4$ & $\pm\left[\sqrt{3}\left(X_{2}+X_{24}\right)+2 i X_{23}\right]$ & 18 \\
12 & 220002 & $6,8,10,14,10,6$ & $\sqrt{6}\left(X_{1}+X_{21}\right)+2\left(X_{2}+X_{24}\right)$ & 10 \\
\hline
\end{tabular}

Table 10.

Cayley triples in $E_{6(-26)}$.

\begin{tabular}{|c|c|c|c|c|}
\hline & $\alpha_{i}(H)$ & $k_{i}$ & $E$ & inv \\
\hline 1 & 100001 & $2,2,3,4,3,2$ & $X_{23}+X_{36}$ & 24 \\
2 & 200002 & $4,4,6,8,6,4$ & $\sqrt{2}\left(X_{1}+X_{6}+X_{29}+X_{31}\right)$ & 24 \\
\hline
\end{tabular}

\section{Appendix.}

In this appendix we give the tables of the signs $\varepsilon(i, j)$ of the structure constants $N(i, j)$ for $1 \leq i, j \leq N$ and for the complex simple Lie algebras $G_{2}$, $F_{4}$, and $E_{6}$. The sign $\varepsilon(i, j)$ occurs in the row $i$ and column $j$. The column numbers are abbreviated: Only the last digit is shown. When $\alpha_{i}+\alpha_{j}$ is not a root, then $\varepsilon(i, j)$ is not defined and we have indicated this by writing a 
zero entry at such positions. In the Tables 12 and 13 we write "p" instead of "+" and "n" instead of "-" (for typographical reasons).

Table 11.

$\varepsilon(i, j)$ for $G_{2}$.

\begin{tabular}{c|cccccc} 
& 1 & 2 & 3 & 4 & 5 & 6 \\
\hline 1 & 0 & + & + & + & 0 & 0 \\
2 & - & 0 & 0 & 0 & + & 0 \\
3 & - & 0 & 0 & - & 0 & 0 \\
4 & - & 0 & + & 0 & 0 & 0 \\
5 & 0 & - & 0 & 0 & 0 & 0 \\
6 & 0 & 0 & 0 & 0 & 0 & 0
\end{tabular}

Table 12.

$\varepsilon(i, j)$ for $F_{4}$.

\begin{tabular}{|c|c|c|c|c|c|}
\hline & 12345 & 67890 & 12345 & 67890 & 1234 \\
\hline 1 & 0p000 & $\mathrm{p} 00 \mathrm{pp}$ & 00p00 & $\mathrm{p} 0000$ & $00 \mathrm{p} 0$ \\
\hline 2 & $\mathrm{n} 0 \mathrm{n} 00$ & 0p000 & $\mathrm{p} 000 \mathrm{p}$ & $00 \mathrm{p} 00$ & $0 \mathrm{p} 00$ \\
\hline 3 & 0p0pp & $\mathrm{p} 0 \mathrm{p} 0 \mathrm{p}$ & 0p000 & 0p00p & p000 \\
\hline 4 & 00n00 & p0pp0 & p0ppp & 0p0p0 & 0000 \\
\hline 5 & 00n00 & 0p0n0 & 00n00 & n0000 & 0p00 \\
\hline 6 & n0nn0 & 0pn00 & $0 \mathrm{n} 00 \mathrm{p}$ & 00p00 & n000 \\
\hline 7 & 0n00n & $\mathrm{n} 0 \mathrm{n} 0 \mathrm{n}$ & 0n0p0 & 0p0p0 & 0000 \\
\hline 8 & 00nn0 & $\mathrm{pp} 00 \mathrm{p}$ & 00n00 & n0000 & n000 \\
\hline 9 & n00np & 00000 & On000 & 00p0p & 0000 \\
\hline 10 & $\mathrm{n} 0 \mathrm{n} 00$ & 0pn00 & $\mathrm{nn00n}$ & 000p0 & 0000 \\
\hline 11 & 0n0n0 & $0000 p$ & 00000 & $\mathrm{n} 000 \mathrm{p}$ & 0000 \\
\hline 12 & 00n00 & $\mathrm{pp} 0 \mathrm{pp}$ & 00p00 & 000p0 & 0000 \\
\hline 13 & n00np & 00p00 & 0n00n & On000 & 0000 \\
\hline 14 & $000 \mathrm{n} 0$ & On000 & 00000 & $\mathrm{n} 0 \mathrm{n} 00$ & 0000 \\
\hline 15 & 0n0n0 & $\mathrm{n} 000 \mathrm{p}$ & 00p00 & 0n000 & 0000 \\
\hline 16 & $\mathrm{n} 000 \mathrm{p}$ & 00p00 & $\mathrm{p} 00 \mathrm{p} 0$ & 00000 & 0000 \\
\hline 17 & 00nn0 & On000 & 00p0p & 00000 & 0000 \\
\hline 18 & On000 & $\mathrm{n} 00 \mathrm{n} 0$ & 000p0 & 00000 & 0000 \\
\hline 19 & 000n0 & 0n00n & 0n000 & 00000 & 0000 \\
\hline 20 & 00n00 & 000n0 & n0000 & 00000 & 0000 \\
\hline 21 & 00n00 & p0p00 & 00000 & 00000 & 0000 \\
\hline 22 & 0n00n & 00000 & 00000 & 00000 & 0000 \\
\hline 23 & n0000 & 00000 & 00000 & 00000 & 0000 \\
\hline 24 & 00000 & 00000 & 00000 & 00000 & 0000 \\
\hline
\end{tabular}


Table 13.

$\varepsilon(i, j)$ for $E_{6}$.

\begin{tabular}{|c|c|c|c|c|c|c|c|}
\hline & 12345 & 67890 & 12345 & 67890 & 12345 & 67890 & 123456 \\
\hline 1 & 00p00 & 000p0 & 00p0p & 000p0 & p00pp & 00p00 & p00000 \\
\hline 2 & 000n0 & 000pp & $0 \mathrm{p} 00 \mathrm{p}$ & $\mathrm{p} 0 \mathrm{p} 00$ & $\mathrm{p} 0 \mathrm{p} 00$ & 00000 & 0000p0 \\
\hline 3 & n00n0 & $00 \mathrm{p} 0 \mathrm{p}$ & 000p0 & $\mathrm{p} 000 \mathrm{p}$ & 00000 & $\mathrm{p} 000 \mathrm{p}$ & 00p000 \\
\hline 4 & 0pp0p & 0p000 & p0000 & 000p0 & $0 \mathrm{p} 00 \mathrm{p}$ & 0p000 & 000p00 \\
\hline 5 & 000n0 & n0pp0 & 0pp00 & 0p000 & 00000 & 00p0p & 0p0000 \\
\hline 6 & $0000 p$ & $0000 p$ & 000pp & 00pp0 & 0p0p0 & $\mathrm{p} 00 \mathrm{p} 0$ & 000000 \\
\hline 7 & 000n0 & 00p0p & 000p0 & $\mathrm{p} 000 \mathrm{p}$ & 000n0 & 00n00 & n00000 \\
\hline 8 & 00n0n & On000 & n000p & 00p00 & p0p00 & 00000 & 000n00 \\
\hline 9 & nn00n & 00000 & n00p0 & 0000p & 0n000 & On000 & 00p000 \\
\hline 10 & 0nn00 & nn000 & 00p00 & 0p000 & 0000n & On000 & 0p0000 \\
\hline 11 & 000n0 & 00pp0 & 0pp00 & 0p000 & 000n0 & $\mathrm{n} 00 \mathrm{n} 0$ & 000000 \\
\hline 12 & 0n00n & 00000 & n00p0 & 000pp & 0000p & 00000 & n00000 \\
\hline 13 & n000n & 0000n & n0000 & $\mathrm{n} 0 \mathrm{p} 00$ & 00p00 & 00000 & 00p000 \\
\hline 14 & 00n00 & nn0n0 & On000 & 00000 & p0p00 & 00000 & 0p0000 \\
\hline 15 & nn000 & $\mathrm{n} 0 \mathrm{n} 00$ & 00000 & $0 \mathrm{p} 00 \mathrm{p}$ & 00000 & 0n00n & 000000 \\
\hline 16 & 0nn00 & 0n000 & 00p00 & 0p0p0 & 0p000 & 000n0 & 000000 \\
\hline 17 & 0000n & 0000n & $\mathrm{n} 000 \mathrm{n}$ & n0000 & n0000 & 00000 & n00000 \\
\hline 18 & 0n000 & n0n00 & 00n00 & 0000p & $0000 p$ & 00p00 & 000000 \\
\hline 19 & n00n0 & n0000 & 0n000 & n0000 & 00p00 & 0000n & 000000 \\
\hline 20 & 00n00 & On0n0 & 0n00n & 00n00 & 00000 & 000n0 & 000000 \\
\hline 21 & nn000 & 00n00 & 000n0 & 0p000 & 0p000 & p0000 & 000000 \\
\hline 22 & 000n0 & n00p0 & 00000 & n0000 & n0000 & $00 \mathrm{p} 00$ & 000000 \\
\hline 23 & 0n000 & 00n00 & 00nn0 & 000n0 & 000n0 & 00000 & 000000 \\
\hline 24 & n0000 & np000 & p0000 & 00000 & 00p00 & 0p000 & 000000 \\
\hline 25 & n00n0 & $0000 p$ & On000 & 00n00 & 00000 & p0000 & 000000 \\
\hline 26 & 00n00 & n0000 & $\mathrm{p} 0000$ & 00000 & $\mathrm{n} 000 \mathrm{n}$ & 00000 & 000000 \\
\hline 27 & 000n0 & 000pp & $0000 p$ & 00000 & 000n0 & 00000 & 000000 \\
\hline 28 & n000n & 0p000 & 00000 & 00n00 & 0n000 & 00000 & 000000 \\
\hline 29 & 00000 & n0000 & p0000 & p000p & 00000 & 00000 & 000000 \\
\hline 30 & 00n0n & 00000 & $0000 \mathrm{p}$ & 000p0 & 00000 & 00000 & 000000 \\
\hline 31 & n0000 & 0p000 & 0p000 & 0p000 & 00000 & 00000 & 000000 \\
\hline 32 & 0000n & 0000n & 000n0 & 00000 & 00000 & 00000 & 000000 \\
\hline 33 & 00n00 & 000n0 & 00n00 & 00000 & 00000 & 00000 & 000000 \\
\hline 34 & 000n0 & 00p00 & 00000 & 00000 & 00000 & 00000 & 000000 \\
\hline 35 & 0n000 & 00000 & 00000 & 00000 & 00000 & 00000 & 000000 \\
\hline 36 & 00000 & 00000 & 00000 & 00000 & 00000 & 00000 & 000000 \\
\hline
\end{tabular}




\section{References}

[1] N. Bourbaki, Groupes et algèbres de Lie, Chap. 4, 5 et 6, Hermann, Paris, 1968.

[2] N. Bourbaki, Groupes et algèbres de Lie, Chap. 7 et 8, Hermann, Paris, 1975.

[3] R. Carter, Finite groups of Lie type: Conjugacy classes and complex characters, J. Wiley, London, 1985.

[4] D.H. Collingwood and W.M. McGovern, Nilpotent orbits in semisimple Lie algebras, Van Nostrand Reinhold, New York, 1992.

[5] D.Ž. Đoković , Proof of a conjecture of Kostant, Trans. Amer. Math. Soc., 302 (1987), 577-585.

[6] Classification of nilpotent elements in simple exceptional real Lie algebras of inner type and description of their centralizers, J. Algebra, 112 (1988), 503-524.

[7] Classification of nilpotent elements in simple real Lie algebras $E_{6(6)}$ and $E_{6(-26)}$ and description of their centralizers, J. Algebra, 116 (1988), 196-207.

[8] - On automorphisms and adjoint orbits of real semisimple Lie algebras, J. Algebra, 94 (1985), 19-29.

[9] - Note on rational points in nilpotent orbits of semisimple groups, Indag. Mathem., to appear.

[10] E.B. Dynkin, Semisimple subalgebras of semisimple Lie algebras, Mat. Sbornik (N.S.), 30 (1953), 349-462; Amer. Math. Soc. Transl., 6 (1957), 111-245.

[11] A.G. Elašvili, The centralizers of nilpotent elements in semisimple Lie algebras, Trudy Tbiliss. Mat. Inst. Razmadze, Akad. Nauk Gruzin., 46 (1975), 109-132 [in Russian].

[12] B. Kostant, Lie group representations on polynomial rings, Amer. J. Math., 85 (1963), 327-404.

[13] B. Kostant and S. Rallis, Orbits and representations associated with symmetric spaces, Amer. J. Math., 93 (1971), 753-809.

[14] J.F. Kurtzke, Centers of centralizers in reductive algebraic groups, Comm. in Algebra, 19 (1991), 3393-3410.

[15] H. Matsumoto, Quelques remarques sur les groupes de Lie algébrique réels, J. Math. Soc. Japan, 16 (1964), 419-446.

[16] J. Sekiguchi, Remarks on nilpotent orbits of a symmetric pair, J. Math. Soc. Japan, 39 (1987), 127-138.

[17] T.A. Springer and R. Steinberg, Conjugacy classes, in 'Seminar on algebraic groups and related finite groups', Part E, Lect. Notes Math., 131 (1969), 167-266, Springer.

Received December 4, 1996 and revised February 19, 1997. This author was supported in part by the NSERC Grant A-5285.

UNIVERSITY OF WATERLOO

WATERLOO, ONTARIO

CANADA N2L 3G1

E-mail address: dragomir@herod.uwaterloo.ca 